\title{
Linear Sensing Response to Ethanol by Indium Oxide Nanoparticle Layers
}

\author{
Vidya N. Singh ${ }^{1}$ and Ganesan Partheepan ${ }^{2}$ \\ ${ }^{1}$ CSIR-National Physical Laboratory, Dr. K. S. Krishnan Marg, New Delhi 110012, India \\ ${ }^{2}$ Department of Civil Engineering, Amity School of Engineering and Technology, Amity University Uttar Pradesh, Noida 201303, India
}

Correspondence should be addressed to Vidya N. Singh; vidyanands@yahoo.com

Received 10 August 2013; Accepted 26 August 2013

Academic Editor: Kalimuthu Kalishwaralal

Copyright (c) 2013 V. N. Singh and G. Partheepan. This is an open access article distributed under the Creative Commons Attribution License, which permits unrestricted use, distribution, and reproduction in any medium, provided the original work is properly cited.

\begin{abstract}
Indium oxide nanoparticles having well-defined particle sizes were synthesized using a chemical capping method. These nanoparticles were used for making the nanoparticle layers without altering the size and morphology of these particles. These nanoparticles and nanoparticle layers were characterized using XRD, TEM, HRTEM and AFM. The ethanol sensing behavior of the nanoparticle layers were studied at different ethanol concentrations. It was observed that the sensor response was linear to the ethanol concentration in the range of 10-1000 ppm. The ethanol sensing behavior has been explained on the basis of the creation of a depletion region due to the adsorbed oxygen and release of the electron in the conduction band in the presence of ethanol (as it takes away the adsorbed oxygen). The explanation has been supported by EDAX results.
\end{abstract}

\section{Introduction}

Indium oxide is a wide bandgap material (bandgap $\sim 3.6 \mathrm{eV}$ ) [1]. It is nonstoichiometric due to the oxygen deficiency, and therefore it is an n-type highly degenerate semiconductor. It has a relatively low resistance $\left(\sim 10^{-4} \Omega \mathrm{cm}\right)$ and has high transmittance in visible region $(\sim 85 \%)$. Due to these properties, it has wide applications in gas sensing towards $\mathrm{O}_{3}$ and $\mathrm{NO}_{x}$. It is also widely used as transparent conducting electrode and electroluminescent devices [2]. It has been shown in most of the reported works that the size of the nanoparticles in the layers/films used for gas sensing is not well defined because of the different methodologies used for making the particles as well as gas sensing layer, and therefore it is difficult to correlate the sensing properties with the size of the particles and other parameters [3]. In this study, the size of the nanoparticles has been preserved in the nanoparticle layers by using a two-step synthesis process. In the first step, nanoparticles having a well-defined particle size have been synthesized by a chemical capping method, and in the second step, the dispersion of the particles has been deposited on the substrates, without altering the size of the particles [4]. For any material to be useful for the gas sensing applications, it is important that its sensor response is linear for a wide range of the gas concentrations [5-8]. In this work, the effect of ethanol concentration on the gas sensing properties of indium oxide nanoparticle layers has been studied without altering the working temperature or the size of the nanoparticles of the nanoparticle layers.

\section{Experimental}

In order to synthesize $\mathrm{In}_{2} \mathrm{O}_{3}$ nanoparticles, $\mathrm{InCl}_{3} \cdot 3 \mathrm{H}_{2} \mathrm{O}$ has been dispersed in ethanol. This dispersion is mixed dropwise in an ammonia solution containing alanine. The mixture was heated for 20 hours at $80^{\circ} \mathrm{C}$ and allowed to cool down to room temperature. Grayish precipitates settle down $[5,8]$. The precipitates were washed several times in deionized water. The washed powder was annealed at $400^{\circ} \mathrm{C}$ at atmospheric pressure. In order to make the nanoparticle layers, these nanoparticles were dispersed in ethanol and ethylene glycol for $24 \mathrm{~h}$. Then the nanoparticle layer was deposited on glass substrates by dip coating for the AFM and XRD studies, and a drop was put on the interdigitated electrodes (DIL16 chip carrier) 
for gas sensing measurements. The substrates were heated at $100^{\circ} \mathrm{C}$ for $1 \mathrm{~h}$ in order to remove the volatile materials.

Structural properties of the nanoparticle layers were investigated using Rigaku X-ray diffractometer (model Giegerflex-D/max-RB-RU200) operating in glancing angle geometry. The X-ray tube was operated at $4 \mathrm{KW}$ power. The diffractograms were recorded at a glancing angle of $2^{\circ}$ for $2 \theta$ values between $10^{\circ}$ and $80^{\circ}$. The data points were recorded at a step size of $0.05^{\circ}$. TEM/HRTEM analysis was carried out using Tecnai G20-stwin $(200 \mathrm{kV})$ high resolution transmission electron microscope. EDAX attached with HRTEM instrument is used for the elemental analysis. Nanoparticles were dispersed ultrasonically in water and spread over carbon coated copper grids for TEM analysis. The surface morphological studies were carried out using Digital Instruments/Nanoscope IIIa atomic force microscope (AFM) operating in contact mode.

For the gas sensing measurements, an external heating arrangement has been used in order to heat the substrate at higher temperatures $\left(400^{\circ} \mathrm{C}\right)$ [9-11]. A thermoelement close to the interdigitated electrodes allows the temperature measurement. The gas-sensing properties in terms of sensor response and dynamic behavior were determined by measuring the time dependent changes in conductance on changing the gas environment in the measurement cell at a temperature of $400^{\circ} \mathrm{C}$.

\section{Results and Discussions}

The X-ray diffraction of $\operatorname{In}_{2} \mathrm{O}_{3}$ nanoparticle sample is shown in Figure 1. All the peaks are due to cubic $\operatorname{In}_{2} \mathrm{O}_{3}$ and are marked in the figure. The broadening of the FWHM is due to the absence of long-range order due to finite size of nanoparticles. The composition of the nanoparticles was determined using EDAX analysis. In the inset of Figure 1, EDAX analysis results are shown. Peaks due to carbon and copper are from the grids used for TEM studies. The In/O atomic $\%$ ratio in EDAX result shows that it is $\operatorname{In}_{2} \mathrm{O}_{3-x}$. Figure 2(a) shows TEM micrograph of the $\operatorname{In}_{2} \mathrm{O}_{3}$ nanoparticle sample. The most probable sizes of the nanoparticles measured using the TEM micrographs are $11 \mathrm{~nm}$. In the inset of Figure 2(a), HRTEM micrograph and electron diffraction pattern of $\mathrm{In}_{2} \mathrm{O}_{3}$ nanoparticles are shown. The lattice spacing measured for $\mathrm{In}_{2} \mathrm{O}_{3}$ nanoparticle is $0.41 \mathrm{~nm}$ which corresponds to (211) plane of cubic indium oxide. The electron diffraction pattern indicates the polycrystalline nature of $\mathrm{In}_{2} \mathrm{O}_{3}$ nanoparticles. The AFM micrograph of Indium oxide nanoparticle layers is shown in Figure 2(b). The micrographs show very fine particles uniformly covering the substrate. The uniform deposition and connectivity between the particles can be qualitatively seen.

A typical gas sensing response curve for the $\mathrm{In}_{2} \mathrm{O}_{3}$ nanoparticle sample has been shown in Figure 3(a). In Figure 3(a), "sensing on" refers to the inclusion of ethanol in the artificial air, and the "sensing off" refers to the closing of the ethanol supply. The sensor response is defined as the ratio of the conductance after inclusion of ethanol in the artificial air to the conductance in artificial air. The gas sensing properties

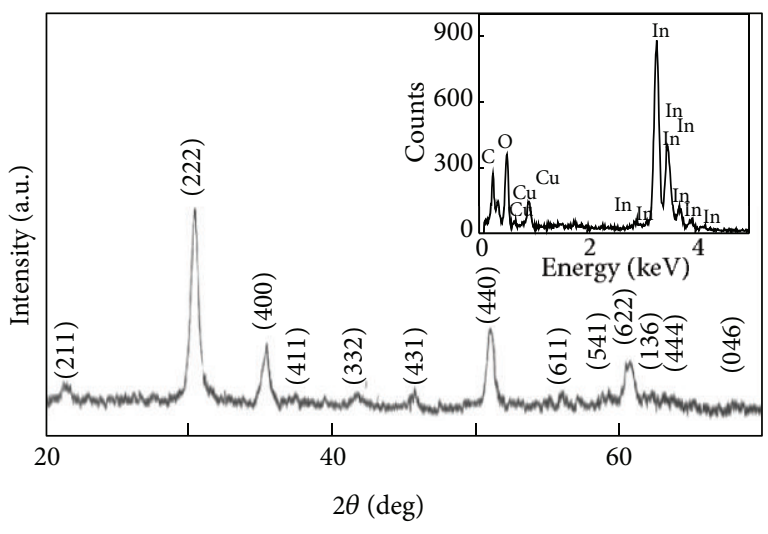

FIGURE 1: GAXRD spectrum of indium oxide nanoparticle layer. All the peaks marked are of cubic $\operatorname{In}_{2} \mathrm{O}_{3}$. Inset shows the EDAX analysis of the nanoparticle sample which confirms it to be oxygen deficient.

have been studied by varying the ethanol concentration. The setup used for studying the gas-sensing properties of the $\mathrm{In}_{2} \mathrm{O}_{3}$ nanoparticle layers has been described elsewhere [911]. The gas-sensing properties in terms of sensor response and dynamic behaviors were determined by measuring the time dependent changes in conductance on changing the gas environment in the measurement cell at a substrate temperature of $400^{\circ} \mathrm{C}$.

Sensor response for $\mathrm{In}_{2} \mathrm{O}_{3}$ nanoparticle samples with ethanol concentration is shown in Figure 3(b). The sensitivity is about 11, 65, 231, and 415 for 10, 100, 500, and $1000 \mathrm{ppm}$ of ethanol, respectively. Thus, a substantial improvement in the performance of the sensor is observed with an increase in ethanol concentration. The plot is a straight line for the ethanol concentration range from 10 to $1000 \mathrm{ppm}$.

It is known that stoichiometric indium oxide is an insulator. But, due to oxygen deficiency, excess indium metal content contributes to the conduction of electrons resulting in a higher electrical conductance. In air, the surface of the indium oxide gets covered with chemisorbed oxygen ions. Adsorbed oxygen at the surface creates a space charge region near the oxide surface by extracting free electrons from the conduction band of oxide, thus decreasing the electrical conductance as shown in Figure 4(a). In the presence of a reducing agent, these oxygen species are removed, and the original conductance is achieved as the electrons captured by oxygen ions are released back to the conduction band of indium oxide as shown in Figure 4(b).

The higher sensor response in the present study is due to the nanoparticles nature of the layer. In our previous report, we have shown that nanoparticles in the size range 5-15 nm show a tremendous increase in sensor response because of the relative increase in the ratio of the surface area to the bulk [3]. When the size of the nanoparticles becomes comparable to the electron Debye length of the material, the conduction mechanism is greatly affected by the environment. In the case of nanoparticle layers, a large volume of the nanoparticle layer gets covered by depletion region in the oxidizing atmosphere. In the reducing atmosphere, all the chemisorbed oxygen is removed by reducing gas resulting in the release of 


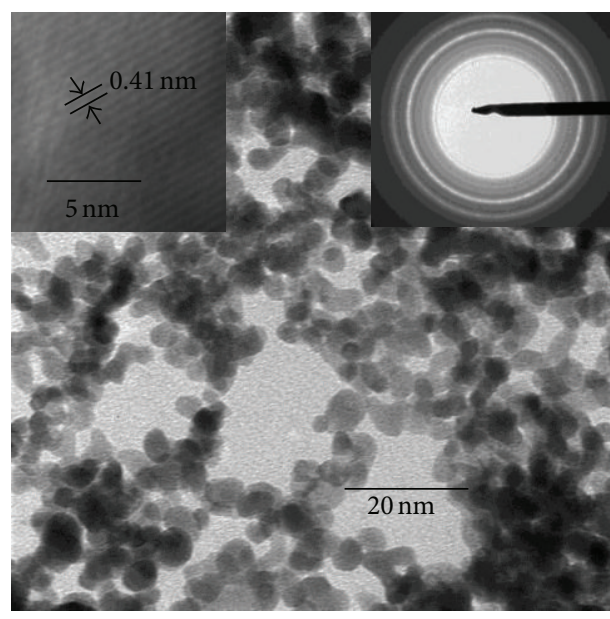

(a)

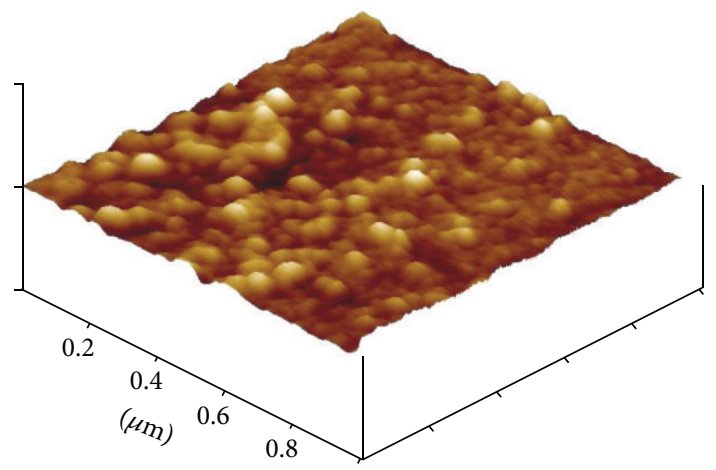

(b)

FIGURE 2: (a) TEM micrograph of indium oxide nanoparticles. The HRTEM micrograph and the diffraction pattern are shown in the inset and (b) AFM image of the indium oxide nanoparticle layers showing the uniformity in the size. It is to be noted that the particles are well connected which is required for the gas-sensing applications.

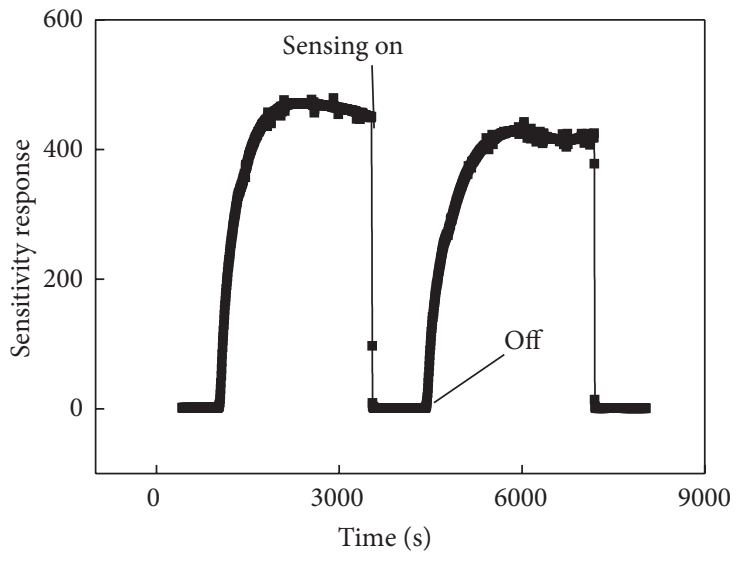

(a)

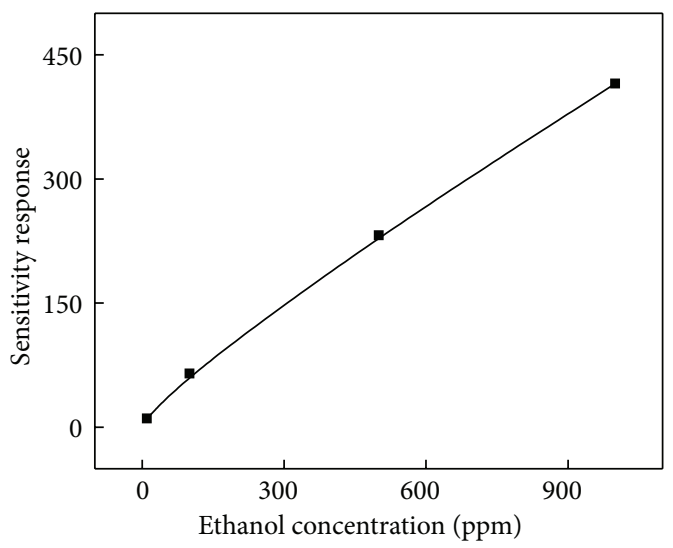

(b)

FIGURE 3: (a) A typical ethanol sensing response of the indium oxide nanoparticle layers; (b) concentration dependent ethanol sensing response of indium oxide nanoparticle layers.

the electrons and thus increased conductance of the layer. Thus, in the case of nanoparticles having size approximately twice the size of the electron Debye length, the sensor response is higher.

When sensor is exposed to the air, $\mathrm{O}_{2}$ gets adsorbed on its surface. The adsorbed oxygen gets translated into the chemisorbed oxygen at a definite temperature

$$
\mathrm{O}_{2}+2 \mathrm{e}^{-} \longrightarrow 2 \mathrm{O}^{-\mathrm{ads}}
$$

Interaction between $\mathrm{C}_{2} \mathrm{H}_{5} \mathrm{OH}$ gas and the chemisorbed oxygen can take place by the following reaction:

$$
\mathrm{C}_{2} \mathrm{H}_{5} \mathrm{OH}+\mathrm{O}^{- \text {ads }} \longrightarrow \mathrm{CH}_{3} \mathrm{CHO}+\mathrm{H}_{2} \mathrm{O}+\mathrm{e}^{-}
$$

The theory predicts that an increase in the concentration of ethanol will lead to an increase in carrier concentration [12]. If we denote the concentration of carriers as $n$, then we can write the following expression for the electrical conductivity of the material [13]:

$$
\Delta \sigma=q \mu_{n} n \propto[X]^{r}, \quad \text { where } 0.5<r>1,
$$

$\mu_{n}$ is mobility of the carrier, $q$ is electron charge, and $X$ is reducing gas concentration.

The relationship between the sensor response and ethanol concentration follows the above formula. Therefore, at lower ethanol concentration (in the range of $10-1000 \mathrm{ppm}$ ), the sensor response increases nearly linearly with the ethanol concentration. Thus, $r$ is found to be 1 . It is to be noted that, in the present study, humidity can not affect the performance as the experiments have been carried out at higher temperature. Also since the working temperature and the particle size in the nanoparticle layers have been preserved; therefore, any change in conductivity is mainly related to the specific gas concentration which affects the electron concentration and 


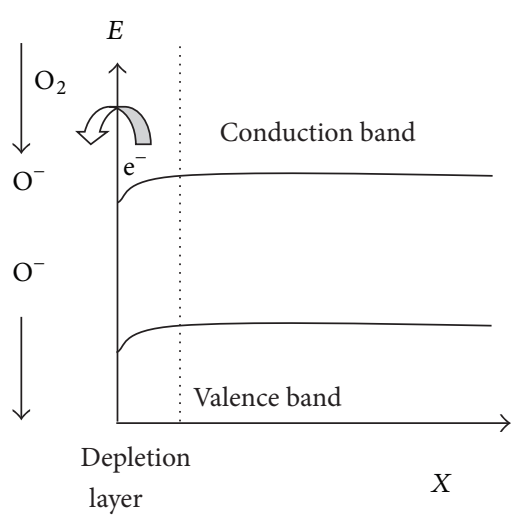

(a)

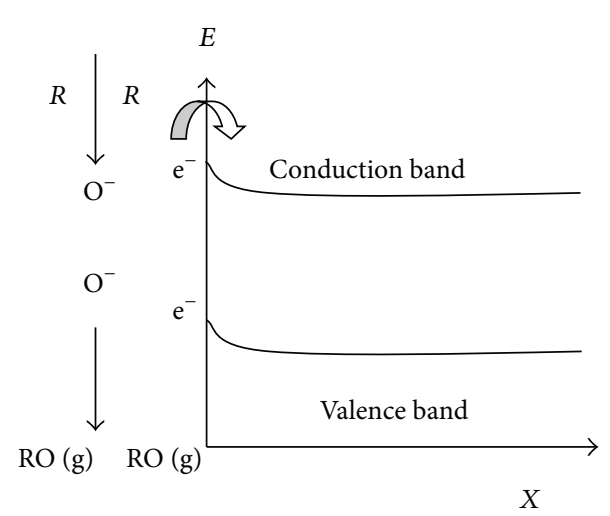

(b)

Figure 4: (a) Oxygen is physisorbed or chemisorbed onto indium oxide surface and takes away an electron from the conduction band, thus creating a depletion layer. This decreases the conductance of the indium oxide nanoparticle layer, and (b) in the presence of a reducing molecule the adsorbed oxygen is removed from the surface; this releases an electron into the conduction layer. This increases the conductance of the indium oxide nanoparticle layer.

electron mobility. At higher concentration, the reaction is faster as the available reducing agents which can take away the oxygen are higher in number.

\section{Conclusions}

In this paper, ethanol concentration dependent gas sensing properties of indium oxide nanoparticle layer have been reported. It has been observed that, for an indium oxide nanoparticle layer, the sensing response of ethanol depends linearly on its concentration in the artificial air. The results have been corroborated with XRD, EDX, TEM, and AFM. This has been explained on the basis of adsorption and desorption of adsorbed oxygen in the air and in the presence of ethanol. The particle sizes in the nanoparticle layer and operating temperature have been kept constant during the measurements.

\section{Conflict of Interests}

The authors declare that they have no conflict of interests.

\section{Acknowledgment}

Vidya N. Singh acknowledges the supports received from Professor B.R Mehta, IIT Delhi, in conducting most of this work.

\section{References}

[1] H. Jia, Y. Zhang, X. Chen et al., "Efficient field emission from single crystalline indium oxide pyramids," Applied Physics Letters, vol. 82, no. 23, pp. 4146-4148, 2003.

[2] M. Marezio, "Refinement of the crystal structure of $\operatorname{In}_{2} \mathrm{O}_{3}$ at two wavelengths," Acta Crystallographica, vol. 20, part 6, pp. 723$728,1966$.

[3] V. N. Singh, B. R. Mehta, R. K. Joshi, and F. E. Kruis, "Sizedependent gas sensing properties of indium oxide nanoparticle layers," Journal of Nanoscience and Nanotechnology, vol. 7, no. 6, pp. 1930-1934, 2007.

[4] V. N. Singh and B. R. Mehta, "A two-step synthesis procedure for $\mathrm{In}_{2} \mathrm{O}_{3}$ nanoparticle films having well-defined particle size," Japanese Journal of Applied Physics 1, vol. 42, no. 7, pp. 42264232, 2003.

[5] V. N. Singh and B. R. Mehta, "Nanoparticle size-dependent lowering of temperature for phase transition from $\operatorname{In}(\mathrm{OH})_{3}$ to in $_{2} \mathrm{O}_{3}$," Journal of Nanoscience and Nanotechnology, vol. 5, no. 3, pp. 431-435, 2005.

[6] L. Q. Nguyen, P. Q. Phan, H. N. Duong, C. D. Nguyen, and L. H. Nguyen, "Enhancement of $\mathrm{NH}_{3}$ gas sensitivity at room temperature by carbon nanotube-based sensor coated with Co nanoparticles," Sensors, vol. 13, no. 2, pp. 1754-1762, 2013.

[7] S. Peng, G. Wu, W. Song, and Q. Wang, "Application of flowerlike $\mathrm{ZnO}$ Nanorods gas sensor detecting $\mathrm{SF}_{6}$ decomposition products," Journal of Nanomaterials, vol. 2013, Article ID 135147, 7 pages, 2013.

[8] B. R. Mehta and V. N. Singh, "Structural, electrical and gassensing properties of in $\mathrm{O}_{3}$ : Ag composite nanoparticle layers," Pramana, vol. 65, no. 5, pp. 949-958, 2005.

[9] V. N. Singh, B. R. Mehta, R. K. Joshi, F. E. Kruis, and S. M. Shivaprasad, "Enhanced gas sensing properties of $\operatorname{In}_{2} \mathrm{O}_{3}: \mathrm{Ag}$ composite nanoparticle layers; electronic interaction, size and surface induced effects," Sensors and Actuators B, vol. 125, no. 2, pp. 482-488, 2007.

[10] R. K. Joshi and F. E. Kruis, "Size-selected $\mathrm{SnO}_{1.8}$ : Ag mixed nanoparticle films for ethanol, $\mathrm{CO}$, and $\mathrm{CH}_{4}$ detection," Journal of Nanomaterials, vol. 2007, Article ID 67072, 5 pages, 2007.

[11] V. N. Singh, B. Raj Mehta, R. K. Joshi, and F. E. Kruis, "Effect of silver addition on the ethanol-sensing properties of indium oxide nanoparticle layers: optical absorption study," Journal of Nanomaterials, vol. 2007, Article ID 28031, 5 pages, 2007.

[12] D.-D. Lee, “Environmental gas sensors," IEEE Sensors Journal, vol. 1, no. 3, pp. 214-224, 2001.

[13] D. E. Williams, "Semiconducting oxides as gas-sensitive resistors," Sensors and Actuators B, vol. 57, no. 1-3, pp. 1-16, 1999. 

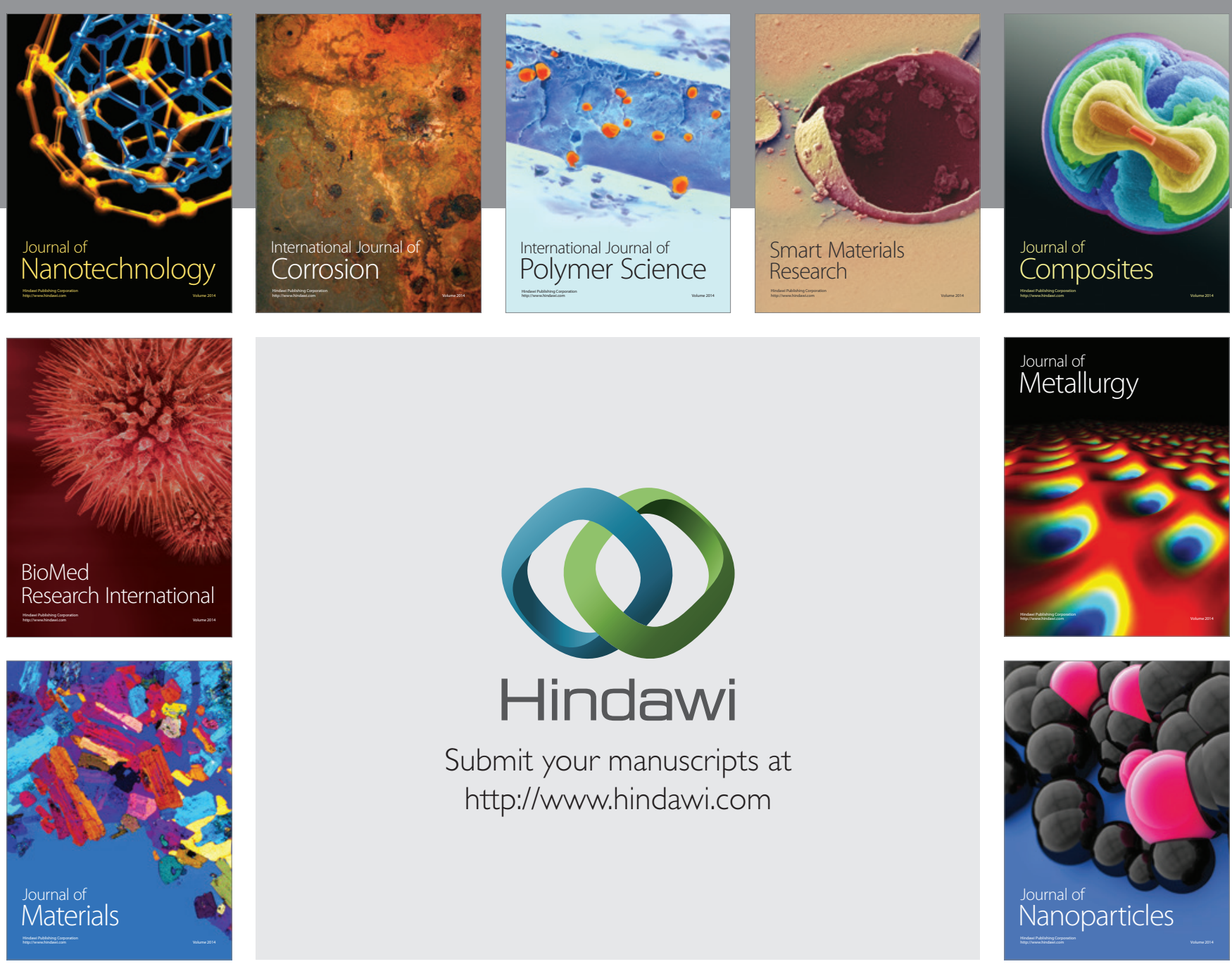

Submit your manuscripts at http://www.hindawi.com
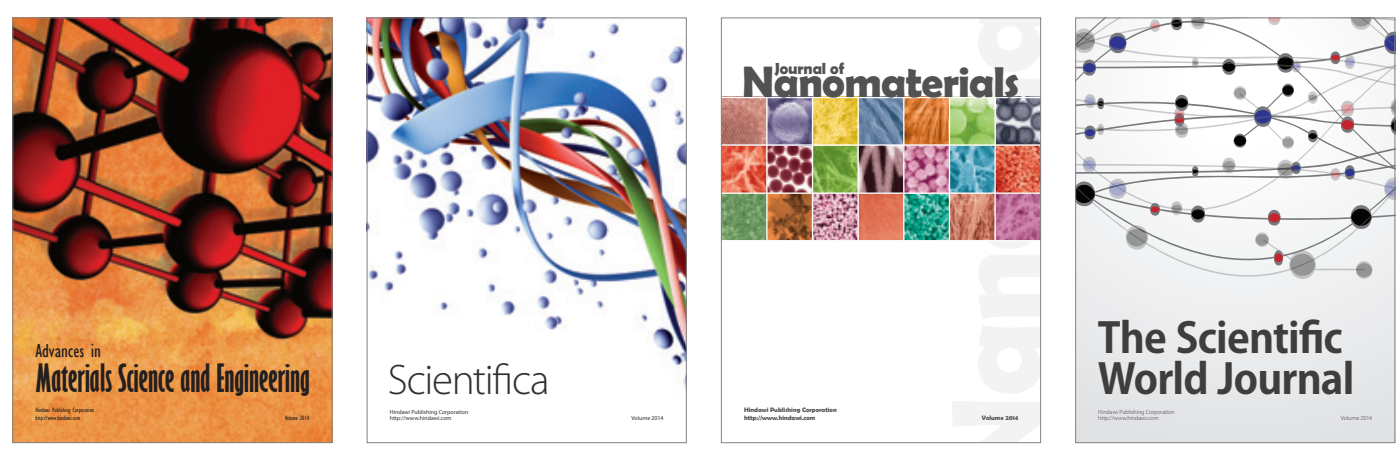

\section{The Scientific World Journal}
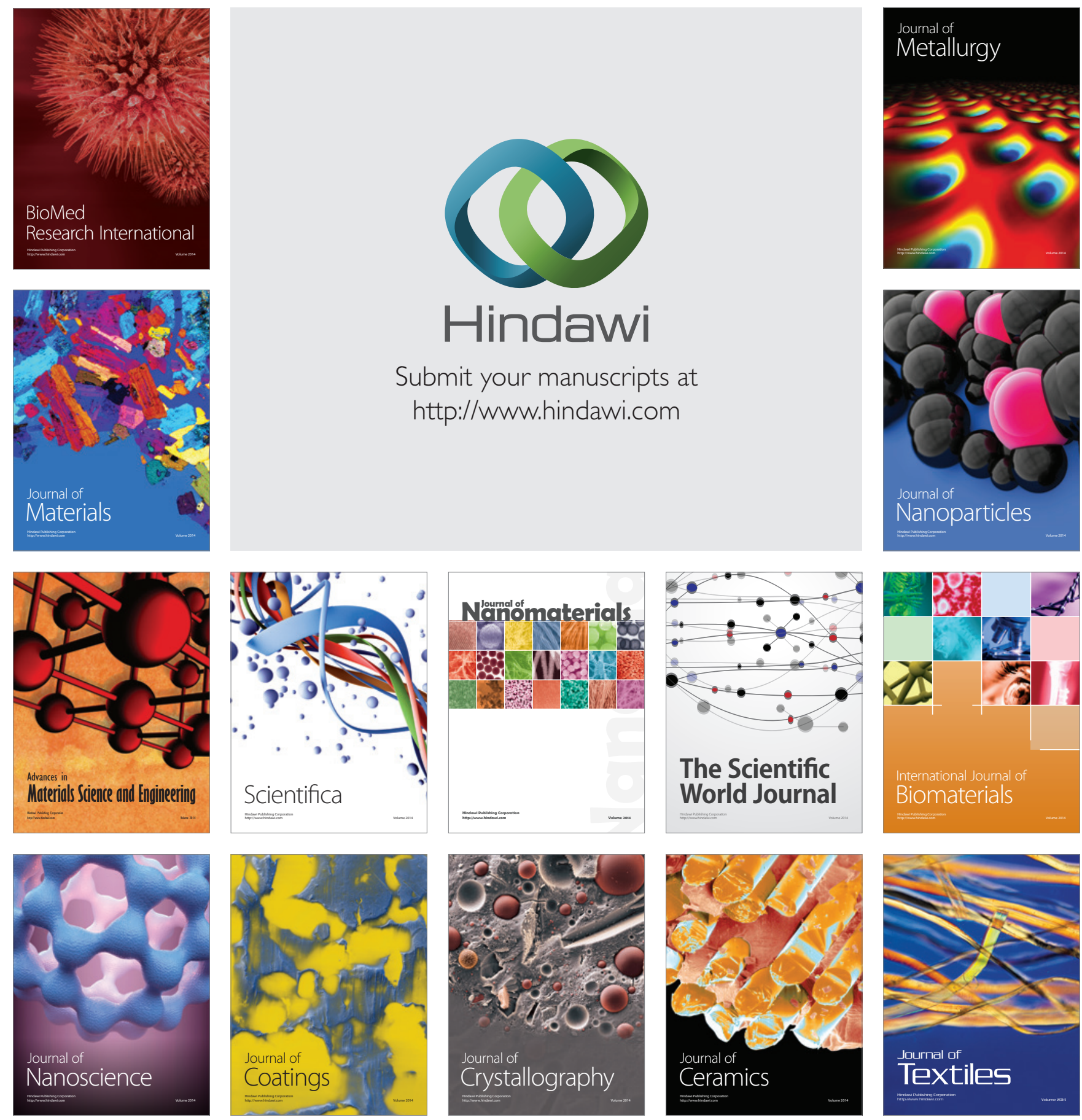